\title{
Design and Simulation of Microstrip Comb-Line Array Antenna at 78.5GHz for Automotive Radar Applications
}

\author{
Chandrakala J, Shanthi P
}

\begin{abstract}
The design of low side lobe level 45 degree linearly polarized microstrip comb-line shaped travelling wave array antenna for $77 \mathrm{GHz}$ automotive radar applications is presented. The 20 elements array is designed on Astra Isola substrate material. Variable width radiating elements are incorporated in the design to utilize an amplitude tapering technique to achieve lower sidelobe level. Taylor distribution is used to determine voltage excitation. The measured gain is found to be around $16.23 \mathrm{~dB}$ and the side lobe level of $-14.28 \mathrm{~dB}$ at $78.5 \mathrm{GHz}$.

Keywords : Microstrip antenna, comb-line array, Automotive Radar, millimeter, Gain, SLL .
\end{abstract}

\section{INTRODUCTION}

Microstrip antennas have a various advantages like low cost, low profile, easy to manufacture etc hence it is most preferred than any other antennas in industry. Microstrip antenna with slots can improve antenna performance and can operate in dual band to enhance the data rate[9]. Single microstrip patch antenna results a poor antenna performance like less gain and directivity. To improve the antenna performance group of similar antennas called array antenna comes into picture. At broadside radiation from each element adds up to produce maximum radiation in a particular direction. Linear polarized travelling wave grid array antenna was proposed by Kraus in 1964. Design of the microstrip array antenna is a very important key factor in a millimeter wave automotive radar. Millimeter wave radars found a wide variety of application in communication systems, automotive industry, 5G[8] which can reduce overall size. Different types of the millimeter wave antennas are used in the automotive industry, in that series feeding microstrip array antenna which is also called as travelling wave array antenna and parallel feeding array antenna are commonly used in $77 \mathrm{GHz}$ automotive radars. Travelling wave microstrip array antenna usually suffers less feeding loss compared to parallel fed antennas [1].

Comb line array antenna consists of slits around radiating elements to nullify the reflected waves from radiating elements [1]. Matching element is placed at feed line terminal

Revised Manuscript Received on July 22, 2020.

* Correspondence Author

Dr. Shanthi P*, Associate Professor, Department of Telecommunication, RV College Of Engineering, Bengaluru, India

Chandrakala J, Department of Telecommunication, RV College of Engineering, Bengaluru, India

(C) The Authors. Published by Blue Eyes Intelligence Engineering and Sciences Publication (BEIESP). This is an open access article under the CC BY-NC-ND license (http://creativecommons.org/licenses/by-nc-nd/4.0/) to obtain better return loss [1] -[3]. Radiating elements are attached to feeding line with a spacing of half guided wavelength in order to excite an elements in-phase [1] [4] [6]. Non-uniform amplitude distribution leads to achieve better side lobe level than uniform amplitude distribution [7]. Higher gain can be achieved using this antenna compare to the series fed antenna when the same PCB area is considered but the lower sidelobe level can be achieved with the series fed antenna.

In order to know the information about the high speed vehicles which are coming in the opposite direction provokes to development of the automotive radar. First radar reacts with the high speed object which is coming in the opposite direction and determines the distance. If the measured distance is found to be crucial i.e., collision cannot be avoided then radar activates the protection system [6].

Modern cars are already equiped with front corner and rear looking automotive radars like millimeter wave radars, narrow band radars. In addition radars can also be installed in all the directions of the car for e.g.,left, right side of front and rear parts and etc to gather the information from all the directions for various functions like measuring distance, blind spot detection and etc.

\section{ANTENNA DESIGN}

Automotive radar frequency considered in this design is $76-81 \mathrm{GHz}$ and the antenna resonates at $78.5 \mathrm{GHz}$. The number of elements considered in an array is 20 and the side lobe level is set around $-20 \mathrm{~dB}$ and the number of side lobes are 5. Non-uniform voltage amplitude distribution results better side lobe level than the uniform amplitude distribution. Taylor distribution is incorporated in this design for voltage excitation distribution. Taylor series is calculated using the equation from (1) to (6):

$R 0=10^{\wedge}(R 0 \mathrm{~dB} / 20)$

$A=(1 / \Pi) x \operatorname{arccosh} R 0$

$A=(1 / \Pi) x\left(R 0+\left(R 0^{\wedge} 2-1\right) 0.5\right)$

$n^{\prime} \geq 2 A^{\wedge} 2+1 / 2$, for $n^{\prime}=2 A^{\wedge} 2+1 / 2+1$ 


\section{Design and Simulation of Microstrip Comb-Line Array Antenna at 78.5GHz for Automotive Radar Applications}

$\sigma=n^{\prime} /\left(A^{\wedge} 2+\left(n^{\prime}-0.5\right)^{\wedge} 2\right)^{\wedge} 0.5$

$$
\begin{gathered}
\ln (z n)=1+2 \sum S^{\prime}(m) \cos (m p) \\
m \quad \forall m=1 \text { to } n^{\prime}-1 \\
n=1 \text { to } N
\end{gathered}
$$

$$
S^{\prime}(m)=S^{\prime}\left(m, A, n^{\prime}, \sigma\right)=S^{\prime}(m, R 0 d B)
$$

The width of antenna elements are obtained as $0.171 \mathrm{~mm}$, $0.160 \mathrm{~mm}, \quad 0.152 \mathrm{~mm}, 0.161 \mathrm{~mm}, 0.183 \mathrm{~mm}, 0.214 \mathrm{~mm}$, $0.224 \mathrm{~mm}, 0.2445 \mathrm{~mm}, 0.256 \mathrm{~mm}$ and $0.253 \mathrm{~mm}$ placed symmetrically with respect to centre. Values are calculated using MATLAB.

\section{ANTENNA STRUCTURE AND SIMULATED RESULTS}

Microstrip comb line array consists of rectangle shaped radiating elements connected to linear feeding line with 45 degree inclined, on both the sides in an interleave fashion on astra isola substrate material having a dielectric constant of 2.925 and thickness of $0.127 \mathrm{~mm}$ beneath the ground plane as shown in Fig.1. Travelling wave Microstrip feed line having a characteristic impedance of $50 \mathrm{Ohm}$ and width of $0.32 \mathrm{~mm}$. Length of radiating elements are approximately half the guided wavelength. Angle between the radiating elements and the feed line is maintained around 45 degree as shown in Fig.1. so that polarizations of radar signals received from vehicles coming in opposite direction are perpendicular to each other hence minimum interference is achieved. Maximum beam direction will be perpendicular to antenna surface area and will be constant for defined frequency band. The Radiating elements length which is almost equal to half the guided wavelength and width of elements are varied. Elements with the larger width emit larger power.

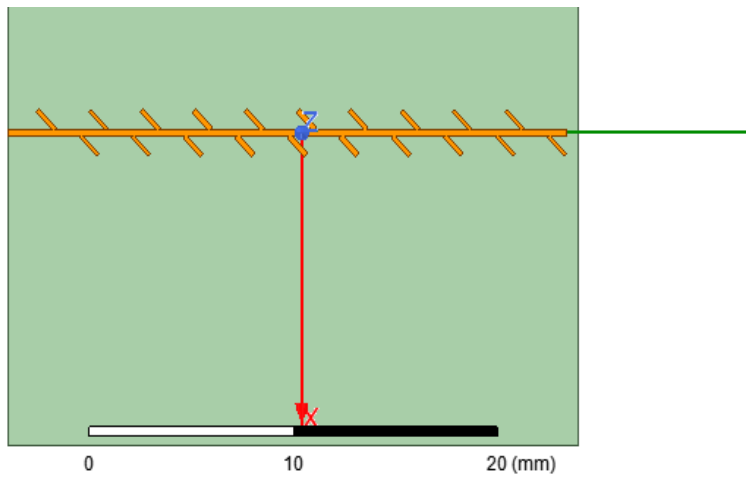

Fig.1. 45-degree polarized microstrip comb line array antenna.

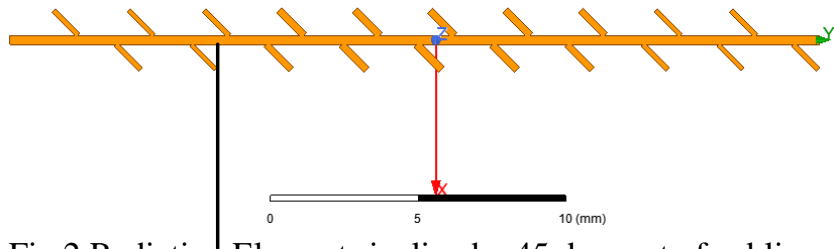

Fig.2 Radiatin Elements incline by 45 degree to feed line.

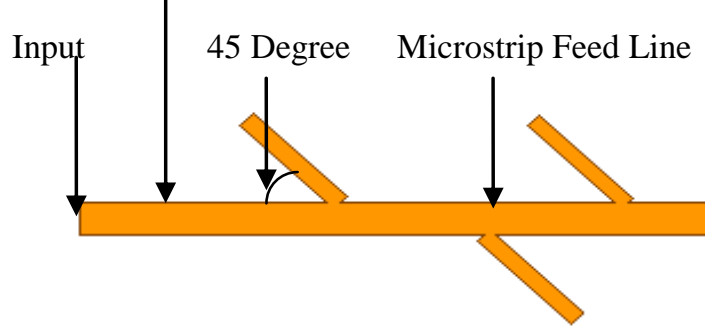

The simulated design results a return loss of $33.26 \mathrm{~dB}$ at $78.5 \mathrm{GHz}$ as shown in Fig.4.Designed microstrip comb line array antenna results a gain of $16.24 \mathrm{~dB}$ and sidelobe level of -14.28dB as shown in Fig.3. 2D Radiation Pattern of an array antenna is as shown in Fig.5.The bandwidth below -10dB is found to be $0.841 \mathrm{GHz}$ as shown in Fig. 4 .

Table.1. Output parameters of microstrip comb-line array antenna.

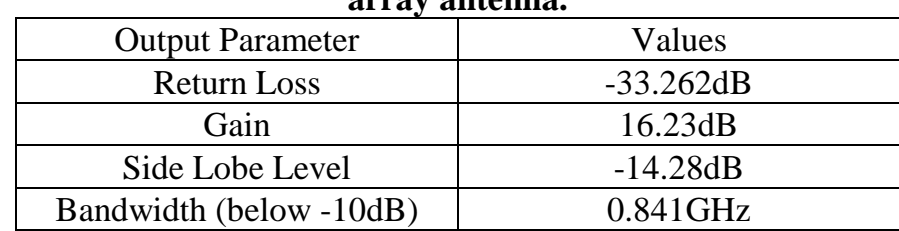




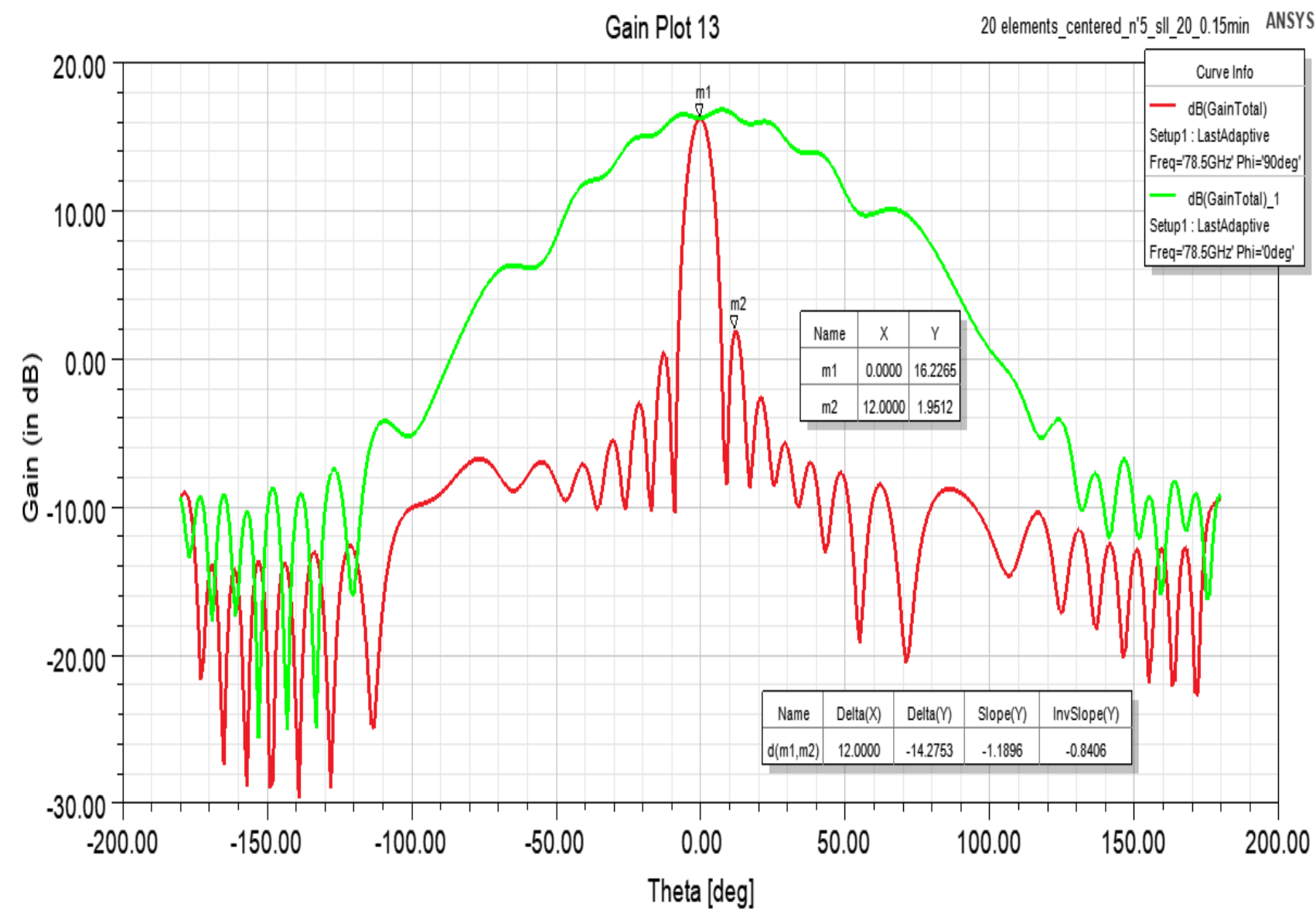

Fig.3.Simulation Pattern of 20 array elements at 78.5 GHz.

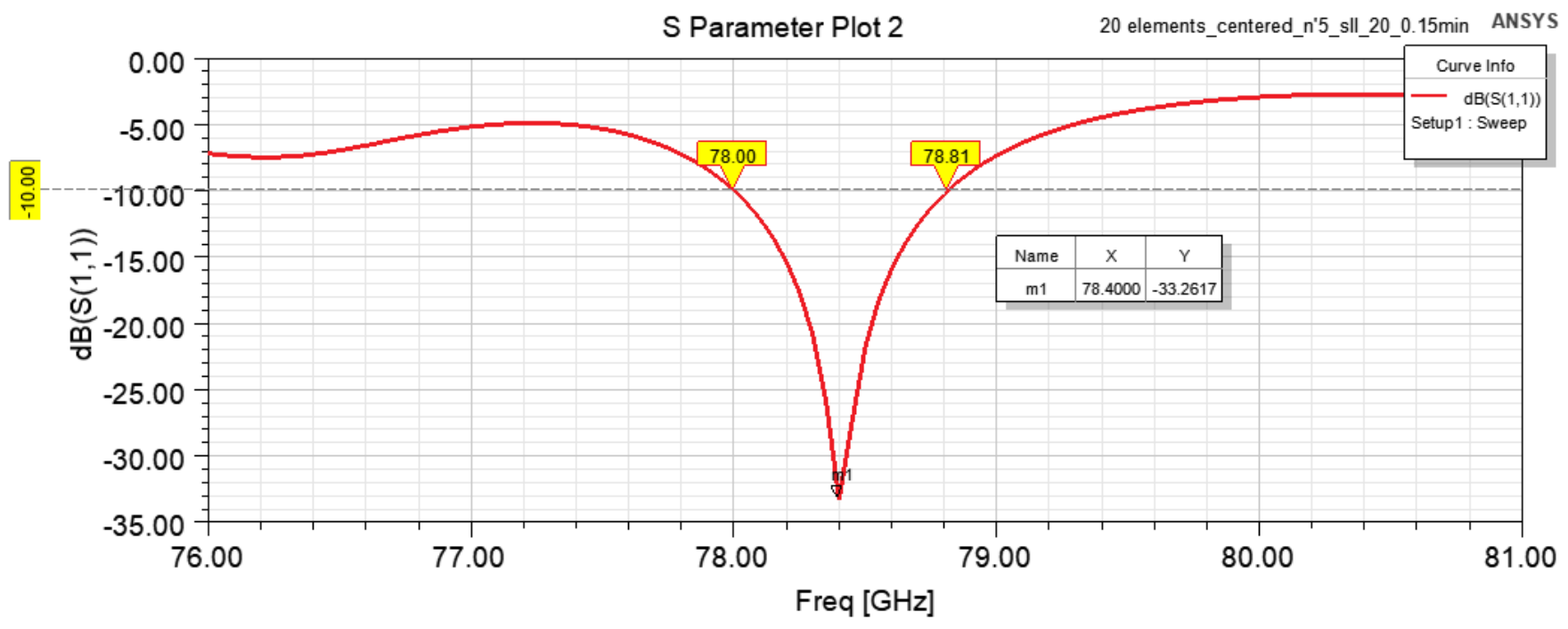

Fig.4. Return Loss of an antenna in 76-81GHz frequency range.

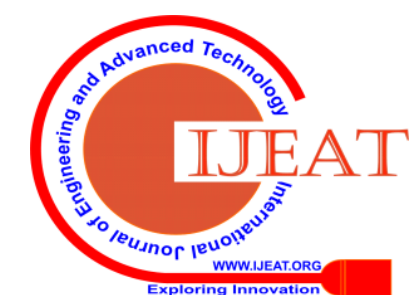




\section{Design and Simulation of Microstrip Comb-Line Array Antenna at 78.5GHz for Automotive Radar Applications}

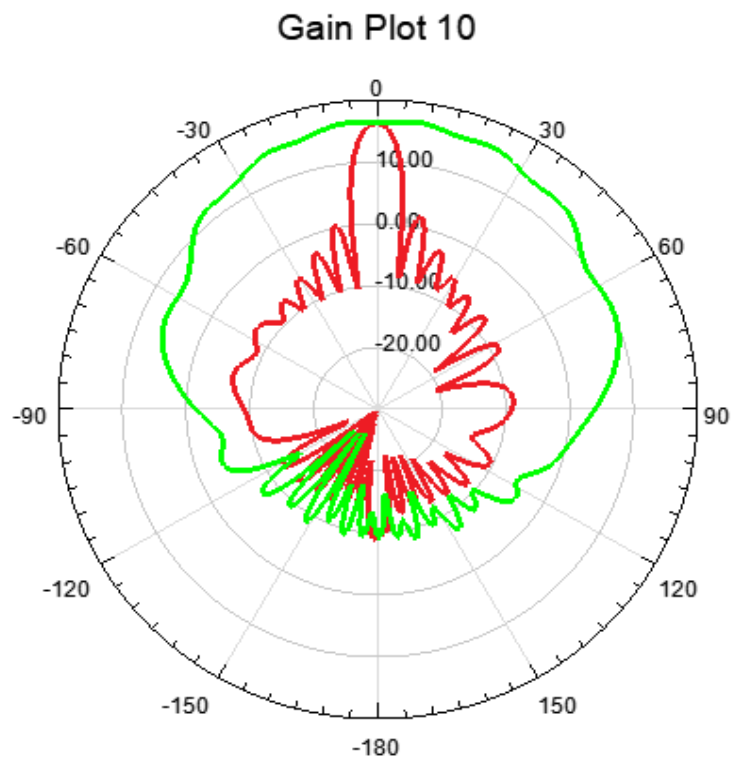

20 elements_centered_n'5_sll_20_0.15min ANSYS

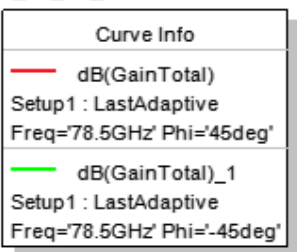

Fig.5. 2D Radiation Pattern of an array antenna.

\section{CONCLUSION}

In this paper, 20 elements microstrip comb line array antenna design for automotive radar applications is presented. The radiating elements are in contact with linear feeding line with 45 degree tilted to achieve polarization required for automotive radar applications. Amplitude tapering technique is employed to achieve better side lobe level. Voltage amplitude excitation of the radiating element can be calculated using Taylor method to achieve better side lobe level. Higher side lobe level results in false target detection. It is 45-degree polarized so that the signals received from the opposite target are orthogonal to each other hence interference is minimized.

\section{REFERENCES}

1. Kunio Sakakibara, Nobuyoshi Kikuma and Hiroshi Hirayama , "Array design techniques of microstrip comb-line antennas in millimeter-wave band ", IEEE Asia Pacific conference on Antennas and Propogation, August. 2012.

2. Kojima, R., Sakakibara, K., \& Kikuma, N, "Traveling-Wave Array Design of Microstrip Comb-Line Antenna for Arbitrary Linear Polarization Using Rounded Radiating Elements", IEEE International Workshop on Electromagnetics: Applications and Student Innovation Competition (iWEM), 2018.

3. Yuki Hayashi, Kunio Sakakibara, Senior Member, IEEE, Morihiko Nanjo, Shingo Sugawa and Nobuyoshi Kikuma. "Millimeter-wave microstrip comb-Line antenna using reflection-canceling slit structure", IEEE transactions on antennas and propagation, vol. 59, no. 2, February 2011.

4. Dapeng Wu, Ziqiang Tong, R. Reuter, H. Gulan and J. Yang, "A $76.5 \mathrm{GHz}$ microstrip comb-line antenna array for automotive radar system," 9th European Conference on Antennas and Propagation (EuCAP), Lisbon, 2015, pp. 1-3.

5. Mohammad Mosalanejad, Ilja Ocket, CharlotteSoens and Guy A. E. Vandenbosch, "Wideband Compact comb-line antenna array for $79 \mathrm{GHz}$ automotive radar applications", IEEE Antenna and Wireless propagation, 2015.

6. Jiahong Lin, Wenhui Shen and Kang Yang," A Low Sidelobe and Wideband Series Fed Linear Dielectric Resonator Antenna Array", IEEE Antennas and Wireless Propagation Letters vol. 58, no. 12, pp. 3898-3904, 2010.

7. Lin Zhang, Wenmei Zhang and Y. P. Zhang, "Microstrip Grid and Comb Array Antennas," in IEEE Transactions on Antennas and Propagation, vol. 59, no. 11, pp. 4077-4084, Nov. 2011.

8. Shanthi.P ,C. V. Krishna and H. R. Rohit, "Design of Slotted Microstrip Patch Antenna for 5G Communications", CIDCA 2019,Springer- Lecture Notes on Data Engineering and Communications Technologies book series (LNDECT)., vol 46, pp. 134-139, Feb.2020.

9. Shanthi P,Soundarya S and Meghana S, "Design of Dual Band Micro Strip Antenna for 2.4 Ghz and 3.6 Ghz", International Journal of Recent Technology and Engineering (IJRTE)., ISSN: 2277-3878, vol-8 Issue-1, pp.2404-2406, May 2019.

\section{AUTHORS PROFILE}
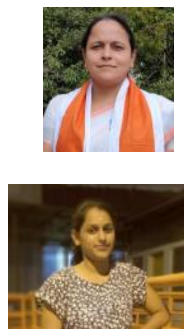

Dr.Shanthi P, Associate Proffessor, Department of Telecommunication, RV College of Engineering, Bengaluru India.

Chandrakala J, Department of Telecommunication, RV College of Engineering, Bengaluru India. 